\title{
Atrial Fibrillation Predicts Worse Long Time Prognosis after CABG-A 6-Year Survival Analysis
}

\section{Lars P. Riber}

Department of Cardiothoracic and Vascular Surgery, Institute of Clinical Medicine, Aarhus University Hospital, Aarhus, Denmark. Email: Larspeterriber@gmail.com

Received January $27^{\text {th }}, 2012$; revised February $29^{\text {th }}, 2012$; accepted March $7^{\text {th }}, 2012$

\begin{abstract}
Background: Postoperative atrial fibrillation occurs in 5\% - 65\% of patients undergoing cardiac surgery. Although postoperative atrial fibrillation is often regarded as a temporary, benign, operation-related problem, it is associated with a two-to threefold increased risk of adverse events, including permanent or transient stroke, acute myocardial infarction, and death. Methods: Two hundred and fifty eligible consecutively enrolled coronary artery bypass grafting patients were included in the randomized, controlled, double-blinded trial RASCABG. That study showed a safe, practical, feasible, and effective prophylactic amiodarone regimen, which significantly decreased the risk of atrial fibrillation with $14 \%$ (5.0 - 24) from $26 \%$ to $11 \%$, with the number needed to treat $6.9(4.2-20)$. This study is a 6 -year follow-up study regarding the long-term prognostic factor of postoperative atrial fibrillation, amiodarone prophylaxis and diabetes mellitus. Results: The long-term 6-year mortality risk of postoperative atrial fibrillation was $31 \%$ equally distributed among patients in the active and in the placebo prophylactic group. The 6-year mortality risk in the sinus rhythm group was $18 \%$ likewise equally distributed, whereas the 6 -year mortality risk in the background population was approximately $8 \%$. The 6 -year mortality risk of diabetes mellitus was $33 \%$ equally distributed, whereas the excluded off pump coronary artery bypass group experiences an increased 6-year mortality risk of $47 \%$. Conclusions: Postoperative prophylaxis with a high dose of oral amiodarone after an intravenous bolus infusion is a safe, practical, feasible, and effective regimen for Coronary Artery Bypass Grafting patients in a 6-year long-term perspective. Atrial fibrillation and diabetes mellitus is correlated to increased mortality risk.
\end{abstract}

Keywords: Atrial Fibrillation; CABG; Pharmacology; Postoperative Care

\section{Introduction}

Postoperative atrial fibrillation occurs in $5 \%-65 \%[1,2]$ of patients undergoing cardiac surgery. The magnitude of risk depends not only on the definition of atrial fibrillation, but also on type of surgery, use of antiarrhythmic medication, and on follow-up time. The latter is the background for this study, which is a 6-year follow-up study of the RASCABG trial [3]. That trial showed a safe, practical, feasible, and effective prophylactic regimen for coronary artery bypass grafting patients. It significantly reduced the risk of atrial fibrillation with $14 \%$ (5.0 - 24) from $26 \%$ to $11 \%$, with the number needed to treat 6.9 (4.2 - 20). The RASCABG trial was based on postoperative oral administrated of a high-dose of amiodarone for five days after an intravenous loading bolus.

In this 6-year follow-up study we hypothesized, that the long-term mortality risk of postoperative atrial fibrillation is increased compared to as well the background population as to the patients in postoperative sinus rhythm. We furthermore hypothesized that diabetes mel- litus is correlated to an increased 6-year mortality risk and that amiodarone is safe to use as prophylactic in the RASCABG setting.

\section{Material and Methods}

Among the 1,500,000 inhabitants of Western Denmark, approximately 1100 persons are referred annually for cardiac surgery to Aarhus University Hospital, Denmark. Two hundred and fifty eligible, consecutively enrolled, coronary artery bypass-grafting patients were enrolled in the randomized, controlled, double-blinded RASCABG trial. In the beginning of the study one patient was drawn randomly each day to be included in the study. After 6 months we shifted the regime to include everybody scheduled for CABG. Each enrolled patient was randomly assigned to receive amiodarone or placebo, based on a computerized prospective randomization schedule. Randomization was done in blocks of four and was stratified by age ( $\leq 65$ years and $>65$ years) and by preoperative use of $\beta$-blockers. 
Each patient received a randomization number, which was recorded and sent by fax to the pharmacy at Aarhus University Hospital, along with the patient's Civil Registration number. The pharmacy decoded the randomization number, prepared the appropriate infusion and pills, and forwarded them, together with a sealed opaque envelope containing the randomization assignment, to the Department of Cardiothoracic and Vascular Surgery.

This study is a 6-year follow-up study regarding the long-term prognostic factor of postoperative atrial fibrillation and diabetes mellitus.

\subsection{Intervention}

In the RASCABG trial coronary artery bypass-grafting patients received a bolus infusion containing $300 \mathrm{mg}$ of amiodarone or placebo (5\% aqueous dextrose solution) over 20 minutes, together with their first $600 \mathrm{mg}$ dose of oral amiodarone or placebo prophylactic in the intensive care unit. Subsequently the oral prophylactic dose of 600 $\mathrm{mg}$ amiodarone/placebo was administered twice a day $(8$ a.m. and 8 p.m.) for five days. Patients in the active treatment group thereby received a total of $6.3 \mathrm{~g}$ amiodarone.

For patients unable to swallow the capsules, a nurse from another department dissolved the tablets in water. The study drug was terminated if adverse effects like drop in systolic blood pressure below $80 \mathrm{mmHg}$, drop in heart rate below 40 beats per minute or other adverse effects were monitored. If possible patients discontinued anti-coagulation treatment 5 days preoperatively and continued the rest of their regular medication, including $\beta$-blockers, until the day of surgery. Medications including $\beta$-blockers were resumed in the intensive care unit on the first postoperative day. During surgery and up until the discharge from the intensive care unit, patients remained under continuous scope monitoring. After transfer to the ward, the attending physician performed standard 12-lead electrocardiograms (ECG) daily for the first five postoperative days. Additional ECGs were obtained in the presence of clinical signs (e.g., irregular pulse, fainting, confusion and shortness of breath) or subjective manifestations (e.g., palpitations, dizziness, fatigue and light-headedness) of atrial fibrillation. If atrial fibrillation developed, the study drug was discontinued, the opaque envelope opened and appropriate treatment was initiated. On postoperative day 30, patients were contacted by a study physician and asked whether they had developed atrial fibrillation. During the same call, patients were asked to contact their physician to schedule an ECG, which was forwarded to the study staff. The observation period ended if the patient reached a study endpoint. All operations were performed via a median sternotomy. A standard cardiopulmonary bypass technique was estab- lished by cannulating the ascending aorta with an aorta cannula and the right atrium with a two-stage catheter. The aorta was cross-clamped, and for myocardial protection cold crystalloid cardioplegia (St. Thomas Solution) was delivered antegrade at twenty-minute intervals throughout the procedure. Noncoated silicone tubes and an oxygenator (QUADROX ${ }^{\circledR}$, Jostra, Hirrlingen, Germany) with an albumin-based coating (Safeline ${ }^{\mathbb{B}}$, Jostra, Hirrlingen, Germany) were used. Conditions of normothermia or moderate hypothermia $\left(34^{\circ} \mathrm{C}\right)$ were established. Bypass conduits included the internal thoracic/mammarian artery, the radial artery, and the saphenous vein. The left internal mammarian artery was anastomosed to the left anterior descending (LAD) in most patients. Distal anastomoses were performed during a single period of aortic cross-clamping, and proximal anastomoses were performed with partial aortic clamping during rewarming.

\subsection{Atrial Fibrillation}

Atrial fibrillation was defined as fast, irregular, eddy current activation of the atrium with neutralisation of its contractions. Electrocardiogram shows totally irregular ventricle rhythm with a constant irregular fluctuation of the length between the QRS-complexes. The QRS-complexes often appear with a frequency around 150 - 200 beats/min, displaying a line flickering with narrow QRScomplexes.

\subsection{Statistical Analysis}

The Pearson's chi-square test was used to compare proportions and to calculate $\mathrm{p}$-values and $95 \%$ confidence intervals. Primary data set was extracted into the Microsoft Excel ${ }^{\circledR}$ software (Microsoft Corporation, United States) and transferred to the Intercooled Stata release 9.0 ${ }^{\circledR}$ statistical package (StataCorp LP, Texas, United States) where all analyses were performed.

\section{Results}

In the RASCABG trial 287 consecutively enrolled patients were randomly chosen as potential participants. 37 were excluded subsequently because of failure to meet inclusion/exclusion criteria $(\mathrm{n}=34)$ or refusal to participate $(\mathrm{n}=3)$. Of the 250 remaining patients intervention was discontinued in 52 patients equally distributed $(\mathrm{p}=$ 0.27). Two-thirds of dropouts were due to surgical related issues as changed operative strategy/off pump surgery or lack of compliance. The postoperative dropout rate was $4.4 \%$.

Baseline data (Table 1) were equally distributed in the two groups except for right coronary artery arteriosclerosis and the median number of grafted vessels, which both 
Table 1. Baseline characteristics.

\begin{tabular}{|c|c|c|}
\hline Characteristic at inclusion & Amiodarone group $\mathrm{n}=125$ & Placebo group $\mathrm{n}=125$ \\
\hline Age in years (minimum, median, maximum) & $39,67,82$ & $35,67,82$ \\
\hline Preoperative use of $\beta$-Blockers, $\%$ & $87 \%$ & $86 \%$ \\
\hline Male, $\%$ & $86 \%$ & $80 \%$ \\
\hline Euroscore (minimum, median, maximum) & $0,3,10$ & $0,2,9$ \\
\hline Hypercholesterolemia, \% & $70 \%$ & $70 \%$ \\
\hline Hypertension, \% & $65 \%$ & $63 \%$ \\
\hline Diabetes mellitus, $\%$ & $22 \%$ & $25 \%$ \\
\hline On insulin & $9.2 \%$ & $10 . \%$ \\
\hline Current smoker, $\%$ & $27 \%$ & $24 \%$ \\
\hline Chronic obstructive pulmonary disease, $\%$ & $9.6 \%$ & $7,2 \%$ \\
\hline Previous myocardial infarction, $\%$ & $50 \%$ & $48 \%$ \\
\hline Ejection fraction (minimum, median, maximum) & $30,57,81^{1}$ & $15,53,81^{2}$ \\
\hline Atrial, mm (minimum, median, maximum) & $23,38,51^{3}$ & $15,40,55^{4}$ \\
\hline Multivessel disease, $\%$ & $100 \%$ & $98 \%$ \\
\hline \multicolumn{3}{|l|}{ Lesion location, $\%$} \\
\hline Left main stenosis & $38 \%$ & $44 \%$ \\
\hline Left anterior descending & $99 \%$ & $97 \%$ \\
\hline Circumflex & $96 \%$ & $94 \%$ \\
\hline Right coronary artery & $97 \%{ }^{\mathrm{a}}$ & $90 \%{ }^{\mathrm{a}}$ \\
\hline
\end{tabular}

${ }^{a}$ The number of grafted right coronary arteries was higher in the amiodarone group (a) $p=0.024 .{ }^{1} 87$ patients; ${ }^{2} 84$ patients; ${ }^{3} 44$ patients; ${ }^{4} 43$ patients.

were overrepresented in the amiodarone group.

Prophylactic amiodarone significantly reduced the risk of atrial fibrillation with a factor 2.3 (1.3 - 4.1). The overall number needed to treat was 6.9 and the risk reduction was $14 \%$ (5.0 - 24), from $26 \%$ to $11 \%$ (Table 2 ). Long-term follow-up after 6 years (Table 3 and Figure 1) showed an independent increased mortality risk of $13 \%$ ( $\mathrm{p}=0.045$ ) from $18 \%$ to $31 \%$ in patients experiencing postoperative atrial fibrillation compared to patients in sinus rhythm throughout the perioperative period. Diabetes mellitus was equally independent correlated to an increased risk of $16 \%(p=0.019)$ from $17 \%$ to $33 \%$ regardless of insulin dependents.

There was found no long-term adverse effects of amiodarone, as 6-year mortality risk of $21 \%$ was equal in the placebo and amiodarone group $(\mathrm{p}=0.704)$. The patients who drop out of the study had the same 6-year mortality risk of $21 \%$ as the patients who completed the study. The exception was the patients who had an off pump coronary bypass performed as they experienced an increased 6 -year mortality risk of $26 \%(p=0.015)$ from $21 \%$ to $47 \%$. The overall 6 -year mortality risk was altogether increased from $8 \%$ in the background population to $21 \%$ in the RASCABG population.

\section{Comment}

In this follow-up study based on the randomized, placebo, controlled RASCABG trial, we found that our regimen of amiodarone administered prophylactic to patients undergoing coronary artery bypass grafting reduced the risk of post-operative atrial fibrillation without increasing the long term mortality risk or adverse outcomes after coronary artery bypass grafting. We found no indication of adverse 6-year long-term outcome for patients in prophylactic regime with $6.3 \mathrm{~g}$ amiodarone administrated over 5 perioperative days, why we therefore consider this prophylactic regime safe.

Postoperative atrial fibrillation and diabetes mellitus were correlated to an increased 6-year mortality risk, why further achievements in reducing the impact and risk of these diseases are recommendable. Therefore a safe prophylactic to avoid the risk of atrial fibrillation is recommendable. 


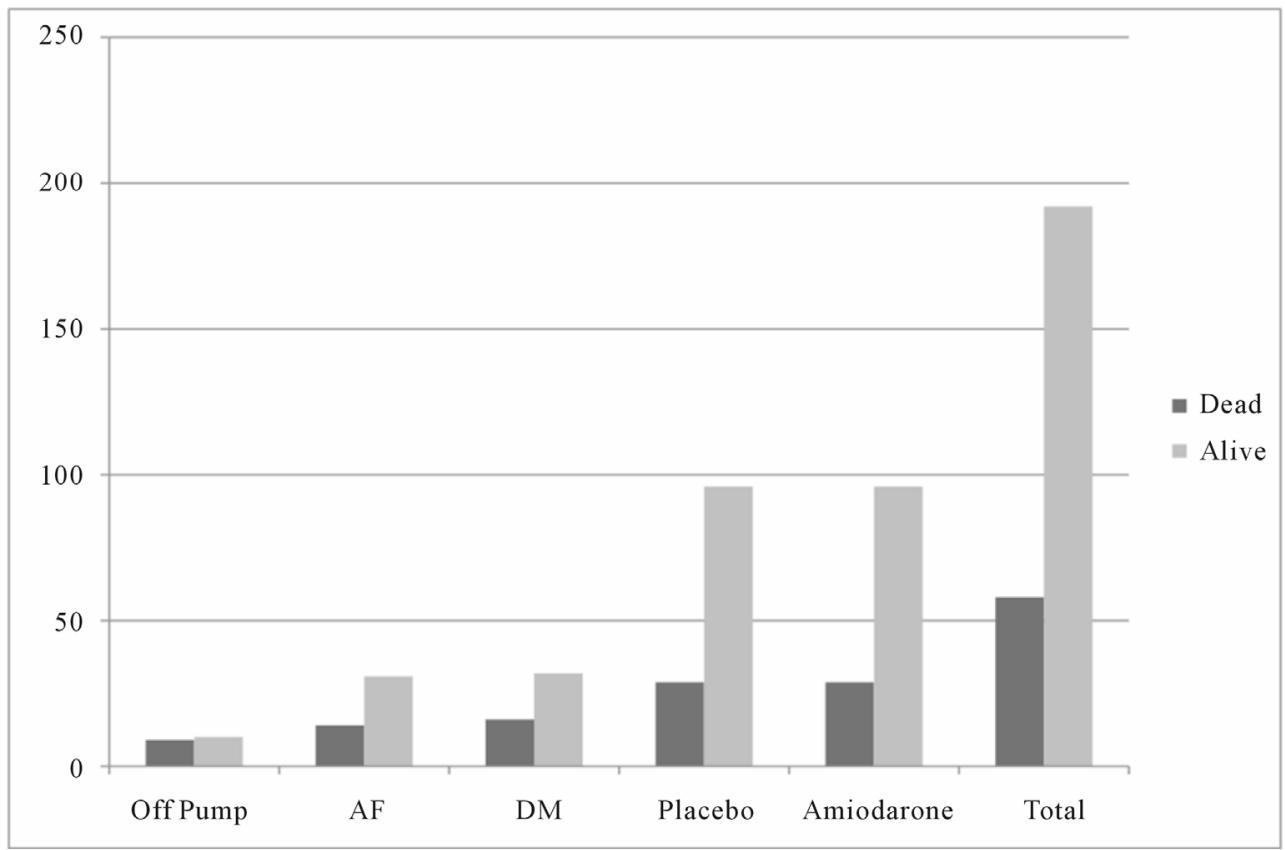

Figure 1. Distribution between deceased and alive.

Table 2. Relative and absolute effects, with numbers needed to treat.

\begin{tabular}{lccccc}
\hline \multicolumn{1}{c}{ Endpoints } & Placebo & Amiodarone & Risk difference $(95 \% \mathrm{CI})$ & NNT $(95 \% \mathrm{CI})$ & Relative risk $(95 \% \mathrm{CI})$ \\
\hline Atrial fibrillation & $26 \%$ & $11 \%$ & $14 \%(5.0-24)$ & $6.9(4.2-20)$ & $2.3(1.3-4.1)$ \\
Symptomatic atrial fibrillation & $22 \%$ & $4.8 \%$ & $18 \%(9.4-26)$ & $5.7(3.9-11)$ & $4.7(2.0-11)$ \\
\hline
\end{tabular}

Table 3. Six year long-term follow-up.

\begin{tabular}{lcc}
\hline \multicolumn{1}{c}{ Patient characteristics } & $\begin{array}{c}\text { Alive } \\
\text { (Number) }\end{array}$ & $\begin{array}{c}\text { Deceased } \\
\text { (Number) }\end{array}$ \\
\hline Prophylactic placebo & 96 & 29 \\
- Without discontinued & 80 & 23 \\
Prophylactic amiodarone & 96 & 29 \\
- Without discontinued & 75 & 19 \\
Discontinued & 37 & 16 \\
- Off pump coronary artery bypass & 10 & 9 \\
- Residual & 27 & 7 \\
Atrial fibrillation & 31 & 14 \\
- Symptomatic & 23 & 9 \\
- Asymptomatic & 8 & 5 \\
- Placebo & 22 & 10 \\
- Amiodarone & 9 & 4 \\
Diabetes mellitus & 32 & 16 \\
- Placebo & 17 & 8 \\
- Amiodarone & 15 & 8 \\
\hline
\end{tabular}

The 6-year mortality risk of $47 \%(p=0.015)$ following off pump coronary artery bypass grafting is though concerning and indicates the need for a large-scale study regarding long-term results of coronary artery bypass grafting versus off pump coronary bypass grafting as the short-term results so far is comparable [4].

\section{Strengths and Limitations}

The strength of this follow-up study is that it is based on a randomized, blinded, controlled design, which was designed to investigate the perioperative risk of atrial fibrillation.

Limitations are correlated to the amount of patients and thereby to the extern validity of this study, as several of the subpopulations are rather small. Therefore the non-significant data may be significant in a larger study and/or the significant data may be insignificant.

This study was furthermore designed as a clinical study, focusing on development of clinical important atrial fibrillation. The real estimate of patients developing atrial fibrillation might therefore be higher. The establishing of the atrial fibrillation diagnosis after hospital discharge required that the patients visited their physician. This could undermine the validity of our data. However, 
our study focused on clinically important atrial fibrillation, ascertained from medical discharge records and from a telephone interview with the treating physician at day 30. At this time, as well as at discharge from hospital all patients had an ECG performed, which was controlled by a study physician, who was blinded to the prophylactic assigned. Therefore the risk of overlooking clinically important attacks is small.

\section{REFERENCES}

[1] S. R. Ommen, J. A. Odell and M. S. Stanton, "Atrial Arrhythmias after Cardiothoracic Surgery," The New England Journal of Medicine, Vol. 336, No. 20, 1997, pp. 1429-1434.

doi:10.1056/NEJM199705153362006
[2] W. H. Maisel, J. D. Rawn and W. G. Stevenson, "Atrial Fibrillation after Cardiac Surgery," Annals of Internal Medicine, Vol. 135, No. 12, 2001, pp. 1061-1073.

[3] L. R. Zebis, T. D. Christensen, H. F. Thomsen, M. M. Mikkelsen, L. Folkersen, H. T. Sørensen and V. E. Hjortdal, "A Practical Regimen for Amiodarone Use in Preventing Postoperative Atrial Fibrillation," The Annals of Thoracic Surgery, Vol. 83, No. 4, 2007, pp. 1326-1331. doi:10.1016/j.athoracsur.2006.09.096

[4] M. E. Halkos, T. A. Vassiliades, J. S. Douglas, D. C. Morris, T. Rab, H. A. Liberman, H. Samady, P. D. Kilgo, R. A. Guyton and J. D. Puskas, "Hybrid Coronary Revascularization versus Off-Pump Coronary Artery Bypass Grafting for the Treatment of Multivessel Coronary Artery Disease," The Annals of Thoracic Surgery, Vol. 92 , No. 5, 2011, pp. 1695-1702.

doi:10.1016/j.athoracsur.2011.05.090 